\title{
Transformación del trabajo y regulación laboral: 1990-2006
}

\author{
Mauricio Lenis Gómez
}

From 1990 onwards, forms of work different to the typical contract employment have become a common feature. Globalization's competitive pressures have brought the trend of labour flexibility and the creation of independent or associative work relationships. The author of this article looks into the relationship between work nature's changes and labour reforms that have been implemented in Colombia since 1990, and subsequently presents the outcomes of these reforms. The article concludes by pointing out the challenges that the transformation of work present to labour legislation.

La década de los noventa en el siglo XX constituye un punto importante de inflexión para analizar las transformaciones en el campo laboral. La mundialización de la economía, que es más visible a partir de dicha década, generó nuevas dinámicas y retos para los empresarios que deben competir en un mercado global. Esta dinámica ha configurado un nuevo modelo de empresa que cambió el contenido y la forma de trabajo, toda vez que organiza la producción conforme a criterios de descentralización basados en la tecnología y la comunicación. Este proceso ha tenido efectos importantes en el trabajo, en los trabajadores y en la regulación laboral.

Precisamente las transformaciones que se dieron en el campo laboral durante la década anteriormente indicada implicó la realización de importantes reformas a la legislación laboral con el fin de estimular la creación de empleo y una mayor cobertura, eficiencia y calidad de servicio en el sistema de seguridad social. Sin embargo, tales reformas no han logrado los resultados esperados.

\footnotetext{
${ }^{1}$ Este artículo recoge las principales reflexiones de la ponencia "Desafíos del derecho laboral frente a la reorganización del trabajo en la empresa", presentada en mayo de 2007 en el XXV Congreso Anual de Derecho del Trabajo, es organizado por el Colegio de Abogados especializados en Derecho del Trabajo y Seguridad Social de Colombia.
} 
Un estudio del BID (2004) indica que el porcentaje de trabajadores que están cubiertos por las normas laborales y los sistemas de protección social es menor hoy que a comienzos de los años noventa. Igualmente, en dicho estudio se señala que los salarios han crecido muy lentamente y que ha aumentado las brechas entre trabajadores más calificados y los no calificados.

La finalidad de este artículo es realizar un análisis de la relación entre los procesos de reorganización del trabajo en la empresa y la legislación laboral, indicando no sólo la forma en que dichos procesos han determinado o influenciado las reformas laborales en las dos últimas décadas, sino también los desafíos que le imponen al derecho laboral. Este análisis implica, por tanto, establecer una relación entre dos variables que determinan el mercado laboral: las sociales y las institucionales (legislación). ${ }^{2}$ Bajo la anterior perspectiva, el eje central de la reflexión será el análisis de las reformas laborales que se produjeron en Colombia en el período 1990-2006, a partir de los procesos de transformación del trabajo en la empresa. Si bien la reorganización del trabajo en la empresa no es un asunto de estudio del derecho laboral, la comprensión de dicho proceso a partir de estudios realizados por otras disciplinas, puede serle de gran utilidad para un mejor desarrollo de su objeto: regular el trabajo.

Este artículo se desarrollará de la siguiente forma. En la primera parte se indica cómo la configuración de un nuevo modelo de organización empresarial cambió el contenido y la forma de trabajo. En la segunda parte se hace referencia a las reformas laborales que se dieron en el período 1990-2006 y sus efectos sobre el mercado laboral colombiano. En la tercera parte se propone una línea de trabajo (problemas y enfoques) que permita establecer diálogos entre diferentes disciplinas para abordar problemas comunes en torno al trabajo. Por último, a manera de conclusiones, se indican los principales retos que afronta el derecho laboral frente a la transformación del trabajo en la empresa.

\footnotetext{
${ }^{2}$ Un mercado laboral es un espacio regido por unas instituciones, en el que se intercambia capacidad productiva entre trabajadores que la ofrecen y personas que la demandan, con el fin de distribuir recursos e ingresos (BID, 2004), y está determinado por múltiples variables, de muy diversos orígenes, entre las que sobresalen las estructurales (determinadas por factores sociodemográficos y los aspectos institucionales que, con carácter de obligatoriedad, regulan el trabajo); las económicas (aquellos factores, internos o externos, que están relacionados con el uso y la distribución de los recursos invertidos y obtenidos de la producción, la distribución y el consumo); y las variables de tipo social (determinadas por las estrategias de organización de la producción y por los modelos de organización del trabajo, cuyo agente es el empresario). El aplicar el concepto de mercado al factor productivo trabajo no significa que a éste último se le adjudique la condición de mercancía.
} 


\section{La reorganización del trabajo en la empresa ${ }^{3}$}

La racionalización del trabajo (intensificación y mejor uso del mismo) ha sido una preocupación constante en el proceso productivo. Desde el proceso de industrialización diferentes modelos han condicionado el proceso y las formas de trabajo en la empresa: taylorismo, fayolismo, fordismo y toyotismo. Para efectos del presente trabajo no se hace necesario caracterizar los diferentes modelos de organización del trabajo, ${ }^{4}$ ni las relaciones de cada modelo con las formas de acumulación de capital, así como tampoco las transformaciones que se han derivado de tales relaciones. Sin embargo, es preciso destacar dos características de estos modelos que son de interés para el presente trabajo. La primera es que los principios de estos modelos se convirtieron en lineamientos internacionales para la organización del trabajo en la empresa. No obstante, asumieron diferentes formas dependiendo de los contextos de cada país. El segundo tiene relación con la incidencia que tales modelos tuvieron sobre la fijación de las normas en los sistemas de regulación laboral. En efecto, los modelos de organización del trabajo de tipo taylorista y fordista coinciden con gran parte de la regulación protectora que se dio en el campo del trabajo hasta buena parte de la década de los 70 en el siglo XX. Los esquemas tradicionales de protección del trabajo dependiente (contratación, jornada de trabajo, salarios, prestaciones sociales, estabilidad laboral, seguridad social, indemnizaciones, sindicalización, entre otros) fueron desarrollados en este período. Esto se debió a que dichos modelos fueron ideados para comprometer al trabajador con las formas de trabajo y con los postulados de la productividad y por lo tanto diferentes beneficios económicos fueron acentuados en la regulación laboral.

\footnotetext{
${ }^{3}$ Es preciso indicar que si bien en este capítulo se hace referencia a un marco general de los procesos de organización empresarial y de la producción en un contexto globalizado, tales procesos de reorganización del trabajo se manifiestan de diferentes formas dependiendo del tipo de actores que los originen (globales, regionales o locales). En este punto se tendrán en cuenta diferentes estudios realizados en el campo de la sociología del trabajo. Entre, otros trabajos, se destacan los siguientes: Castillo, 1998; Castells, 1999, 2001; Beck, 2000; Carnoy, 2001; De la Garza, 2001; Urrea, 2003; Castel, 2004. Para el contexto colombiano, los estudios de Fernando Urrea (2002) y de Fernando Urrea y Carlos Mejía (2000) analizan algunas de las formas que tales procesos asumieron en Colombia. También pueden revisarse los trabajos de Anita Weiss (1994) y de Rainer Dombois y Carmen Marina López (1993).

${ }^{4}$ Bajo el modelo taylorista se presentó un proceso de racionalización del trabajo y de intensificación del mismo (medida de los tiempos y movimientos) que fue utilizado para disciplinar la mano de obra y así establecer un control sobre el trabajador. Esta racionalización estuvo asociada a incentivos económicos otorgados al trabajador para comprometerlo con el postulado de la productividad en la empresa. En el fordismo se continúa con este proceso de racionalización del trabajo y se organiza el trabajo en cadena permitiendo una producción estandarizada. Bajo este modelo se ofrecieron también estímulos económicos y se impuso un control social al trabajador
} 
La organización del trabajo (Novick y Miravalles, 2002) "es el conjunto de aspectos técnicos y sociales intervinientes en la producción de un objeto o servicio. Se refiere a la división del trabajo entre las personas y entre éstas y las máquinas. Es el resultado del conjunto de reglas y normas estandarizadas que determinan cómo se ejecuta la producción y el intercambio de la información y el conocimiento en la empresa". El modelo de organización del trabajo que predomina en el contexto actual bien podría afirmarse que comenzó a gestarse en la década de los años 70 y que se consolidó a partir de los años 90. La década de los 90 en el siglo XX se caracterizó por una fuerte competencia internacional, procesos de integración económica e innovación tecnológica, que a su vez incidieron en una transformación del modelo de acumulación de capital. Los anteriores procesos generaron nuevas estrategias y dinámicas empresariales, impulsadas además por el auge de teorías administrativas que terminaron redimensionando los modelos de organización empresarial y del trabajo. Por auge de teorías administrativas se entiende un ascenso de saberes y conocimientos especializados en torno a la gestión y manejo racional moderno de recursos humanos, materiales y económicos, en el contexto de la producción de bienes y servicios.

Como consecuencia de los anteriores factores, las empresas se reorganizaron en "unidades económicas de menor tamaño, integradas a medios tecnológicos y de comunicación complejos" (Urrea, 2003), basados en criterios de flexibilización, deslocalización y externalización. Por flexibilización se puede entender "la posibilidad de que los cometidos laborales y el tiempo de trabajo puedan adaptarse constantemente a los productos, procesos y mercados cambiantes, lo que hace a los trabajadores más autónomos en el proceso laboral y también a las empresas, que aflojan los lazos que las ligan con los trabajadores" (Carnoy, 2001). Esta flexibilización puede ser interna o externa (Martín, 1995; Urrea, 2003). La interna obedece a organizaciones flexibles en la administración y en la organización de la producción, así como a las formas de contratación de servicios, es decir, a la flexibilización en el uso de la fuerza de trabajo. La externa corresponde a la subcontratación y al uso de la mano de obra temporal. ${ }^{5}$

aún por fuera del trabajo. Las relaciones de trabajo eran jerarquizadas. Bajo el modelo Toyota se da un avance en los procesos productivos como consecuencia de los avances tecnológicos. Asimismo, en este tipo de modelo el trabajador puede desempeñarse en diferentes actividades (polifuncional) que le permite romper con las actividades monótonas y repetitivas que se daban bajo el modelo fordista. Esto permite en cierta medida que las relaciones de trabajo no sean tan jerarquizadas. De otra parte, la producción estandarizada cede ante la producción de acuerdo a las necesidades del cliente y las órdenes de compras, con el fin de controlar los grandes stocks de productos que se dieron bajo el modelo fordista. Ideas como "calidad total" y "justo a tiempo" fueron desarrolladas en el modelo Toyota. Una caracterización ampliamente detallada de estos modelos puede ser consultada en Benjamin Coriat $(2000 ; 2001)$

${ }^{5}$ Debe advertirse que la descentralización de la empresa no siempre implica una ausencia

\section{PRECEDENTE}


La deslocalización por su parte implica "la posibilidad de traslado de los procesos productivos a diferentes partes, de una sede a otra, de un país a otro. Las nuevas unidades productivas con tecnología de punta pueden ser desmanteladas y reubicadas rápidamente" (Capella, 1997). La externalización es la dedicación de la empresa a las actividades que corresponden a su objeto o negocio principal (Urrea, 2003), que implica el desplazamiento de ciertas actividades al sector servicios. Los componentes centrales de los nuevos modelos de organización del trabajo (Novick y otras, 1998) "parten de una combinación heterogénea de las siguientes variables: base tecnológica, flexibilidad del uso de la mano de obra y organización industrial".

Los efectos o resultados de los procesos de reorganización del trabajo en la empresa sobre el trabajo, los trabajadores y la regulación laboral no pueden ser generalizados. Por ejemplo, para algunos como Richard Sennett (2000) el resultado de dicho proceso al presente fue la flexibilización de las prácticas empresariales e institucionales, de tal modo que también el trabajo se redimensiona y adquiere nuevas significaciones tanto para los empleadores como para los sujetos que se mueven en el plano social de los empleados. Los unos y los otros se amoldan a esta nueva racionalidad de modo que los ejes de la vida social laboral se desplazan, y el lenguaje del trabajo se superficializa y permiten, a base de una mayor movilidad, gestiones más productivas y menor gasto de recursos en el proceso productivo. Pero para otros autores como Martín Carnoy (2001), la reorganización de la empresa y del trabajo puede representar el surgimiento de nuevos puestos de trabajos, mayor movilidad y mayores ingresos para el trabajador, en algunos casos. Para la OIT $(2006)^{6}$ el proceso de reorganización del trabajo en la empresa "está ejerciendo una gran influencia en las modalidades del empleo, las condiciones de trabajo, los sistemas de seguridad social y la adaptación de los mecanismos de gobernanza".

No obstante, teniendo presente la anotación anterior, en términos generales podrían sintetizarse de la siguiente manera los efectos de la transformación del modelo productivo en la empresa sobre el trabajo, los trabajadores y la regu-

de responsabilidad laboral. Acorde con la legislación colombiana es posible establecer una responsabilidad solidaria entre la empresa usuaria (que descentraliza) y la empresa contratante, frente a las obligaciones laborales de los trabajadores de esta última, cuando las actividades contratadas hacen parte del giro ordinario de negocios de la empresa usuaria.

${ }^{6}$ Para la Organización Internacional del Trabajo los principales factores de la transformación del trabajo han sido: "el imperativo del desarrollo que se origina en la necesidad de reducir la pobreza y la desigualdad; la transformación tecnológica que entrañó una transformación radical en los medios de producción, distribución e intercambio; y la intensificación de la competencia a nivel mundial tras a liberalización comercial y financiera, así como la reducción de los costos de transporte y comunicación”. 
lación laboral..$^{7}$ En relación con el trabajo, la organización del mismo se basa más en relaciones horizontales que en relaciones jerárquicas. Los empleos que surgen con base en las nuevas tecnologías demandan un trabajador polifuncional, toda vez que el trabajo se basa más en el conocimiento que en actividades físicas. Si bien este avance tecnológico puede implicar la pérdida de puestos de trabajo, también puede posibilitar el auge de otro tipo de trabajos.

En relación con los trabajadores pueden indicarse, entre otros, los siguientes efectos: disminución de los beneficios económicos; aunque en otros casos, dependiendo de las profesiones y de los grados de calificación puede significar aumento de los mismos; liberación del tiempo de trabajo, lo cual puede afectar la relación entre tiempo de trabajo-tiempo libre y las relaciones familiares (Carnoy, 2001). Existe una tendencia al aumento de la carga laboral (Urrea, 2003) y por ende, hay mayor responsabilidad en el trabajador. Sin embargo, no hay un compromiso de la empresa en el tema de la estabilidad laboral, lo que genera una mayor movilidad laboral.

Por otra parte, la reorganización del trabajo ha generado un auge en el uso de otras modalidades o formas de trabajo, como la independiente y la asociativa, frente a la modalidad de trabajo dependiente, la cual era la más visible hasta la década de los 90 en el siglo XX. En efecto, como consecuencia del proceso de transformación del trabajo se evidencia una tendencia en el desplazamiento de las actividades de las empresas hacia el sector servicios, lo que explica la tendencia a excluir a los trabajadores del ámbito de aplicación del derecho laboral, utilizando otras formas de contratación o figuras del derecho civil o comercial. Esto ha debilitado la relación entre trabajador y empleador y se ha venido dando paso a la de contratante o usuario de servicios (trabajador independiente). Para el trabajador tal situación implica un desplazamiento del riesgo en el trabajo ya que él debe financiar la seguridad social como trabajador independiente, y una individualización de los trabajadores frente a la modificación de los vínculos colectivos de trabajo: pérdida de sentido de las asociaciones del tipo de los sindicatos.

Debe recordarse que el trabajo es una noción que abarca toda actividad productiva, de cualquier tipo y con cualquier finalidad concreta (Deveali, 1966). Puede ser definido como todo esfuerzo intencional destinado a modificar la naturaleza o la sociedad. Asimismo, en el sector privado puede ser desarrollado a través de diferentes modalidades: dependiente, independiente, asociativo y voluntario. ${ }^{8}$ El trabajo dependiente consiste en la puesta a disposición de la capacidad laboral y productiva, por parte de un individuo y en beneficio

\footnotetext{
${ }^{7}$ Este aspecto será desarrollado en el punto siguiente.

${ }^{8}$ Para el derecho esta distinción es importante porque no todas las modalidades tienen la misma regulación. Para otras disciplinas, como la economía, esta clasificación no es necesaria porque todas son agrupadas bajo la categoría de empleo.
} 
de otro. Este último, denominado empleador, dirige y remunera la actividad productiva que se va a realizar. Esta modalidad del trabajo se materializa bajo la forma de empleo.

El trabajo independiente, por su parte, se caracteriza por ser una actividad productiva realizada por un individuo y no subordinada a la dirección de otra persona. Esta modalidad de trabajo se materializa de diferentes formas, entre las que se destacan la empresa y la prestación de servicios. El trabajo asociativo es aquella actividad productiva en la que las personas se unen en función de fines y objetivos en común y en la que los asociados esperan beneficiarse de los excedentes generados de su actividad colectiva. Esta modalidad de trabajo se materializa a través de la constitución de organizaciones de trabajo asociado tales como cooperativas de trabajo asociado y empresas asociativas de trabajo. El trabajo voluntario es aquella actividad que se realiza por una decisión de la persona y no por obligación o deber, en función de la producción de bienes o servicios, materiales o intelectuales, y que se caracteriza por responder a fines nobles, valores, aptitudes, actitudes, es decir, por representar para el individuo una ocupación que da sentido a su existencia, y en la que, en consecuencia, el beneficio económico puede pasar a un segundo plano. Esta modalidad puede ser de tipo familiar (oficios domésticos, o ayuda a la empresa familiar sin remuneración), o de tipo voluntariado, en el que la característica esencial es su connotación social. El trabajo voluntario del tipo voluntariado puede obedecer, entre otras, a razones sociales, políticas o religiosas

No obstante, el uso de otras modalidades de trabajo diferentes a la dependiente no siempre implica un aspecto negativo en el campo del trabajo. El cuestionamiento que puede hacerse a algunas modalidades de trabajo (independiente y asociativa, principalmente) consiste en el uso indebido que de ellas se hace en la empresa para disfrazar un contrato de trabajo y así eludir las obligaciones económicas que se derivan del mismo. Es en este sentido que algunos autores critican el uso de algunas modalidades como formas que precarizan el trabajo. Esta situación ha sido analizada, entre otros, por Boltanski y Chiapello (2002) para quienes "al lado del núcleo de quienes aun preservan una relativa protección del empleo, estarían creciendo los segmentos de grupos de trabajadores en los que se acumulan las desventajas generadas por la precarización del trabajo. Este esquema posibilita que las empresas se vuelvan coordinadoras de un proceso productivo sin trabajadores ni máquinas". Es decir, bajo el nuevo esquema de organización del trabajo los grupos de trabajadores que pueden coexistir en la empresa son los siguientes: un pequeño grupo de trabajadores protegidos, a quienes se aplica la legislación laboral; un amplio número de trabajadores que gozan de algún nivel de protección laboral; y otro grupo de trabajadores que hacen parte de los procesos de subcontratación y quienes no gozan de ninguna protección laboral. 
En conclusión, este nuevo modelo de organización en el trabajo ha impulsado el uso de nuevas formas de trabajo que han cambiado su mismo contenido. Ya éste no está asociado exclusivamente al trabajo dependiente y al empleo, forma más visible hasta la década de los años 90 del siglo XX. Asimismo, aspectos como la estabilidad laboral y algunos beneficios económicos que se asociaron a esta modalidad de trabajo se han ido diluyendo. El trabajo en el contexto actual se caracteriza por la flexibilización, la movilidad y la especialización.

\section{Reformas laborales en Colombia: 1990-2006}

Del capítulo anterior se desprende que el contexto económico que caracterizó la década de los 90 del siglo XX y los procesos de reorganización empresarial posibilitaron "una evolución del pensamiento político hacia una mayor confianza en el papel de los mercados y la disminución de la acción del Estado" (OIT, 2006). En este capítulo se hará una referencia a la evidencia de la tendencia hacia la desregulación o la flexibilización de las relaciones laborales. La desregulación consiste en eliminar una obligación establecida para los sujetos de la relación laboral. Por flexibilización de las normas laborales se entiende una disminución de las obligaciones o de los costos económicos establecidos para los mismos sujetos. Por lo general la desregulación o la flexibilización favorecen a la empresa en la medida en que se eliminan obligaciones de diversa índole en relación con los contratos, o se minimizan las mismas.

En Colombia, al igual que en la mayoría de países latinoamericanos (Guasch, 1998), en el período analizado se llevaron a cabo importantes reformas laborales, las cuales fueron incorporadas a través de las siguientes normas: Leyes 50 de 1990, 100 de 1993 y 789 de 2002. Vinculadas a la ley 100 de 1993, entre otras normas, se expidieron las leyes 776 de 2002, 797 de 2003, 828 de 2003 y 860 de 2003. Por otra parte, el acto legislativo 1 de 2005 introdujo importantes cambios en el régimen pensional. En general, se realizaron cambios en materia individual de trabajo, en derecho colectivo y en seguridad social. Las leyes aprobadas establecieron las siguientes reformas. La ley 50 de 1990 flexibilizó los regímenes de contratación, de despidos y los mecanismos de fijación de los salarios, prestaciones sociales y jornada de trabajo. Aumentó la tabla de indemnizaciones para despido sin justa causa en el contrato a término indefinido. Eliminó la retroactividad de las cesantías. Aumentó la protección a la maternidad. Modificó la contratación temporal y algunas normas colectivas de trabajo (facultad de constituir sindicatos sin permiso previo, personería jurídica, eliminación de la posibilidad de suspensión por vía administrativa de la personería jurídica de los sindicatos).

\section{PRECEDENTE}


La ley 100 de 1993 reformó todo el sistema de seguridad social y lo reorganizó en tres subsistemas: pensiones, salud y riesgos profesionales. Eliminó el monopolio del Estado en la prestación de los servicios que ofrece el sistema y se estableció un modelo de competencia regulada. Realizo ajustes a los aspectos financieros del sistema de seguridad social. Contrarrestó la dispersión de regímenes dentro del sector público y estableció una política de cobertura universal. Por su parte, la ley 789 de 2002 flexibilizó la regulación sobre la jornada de trabajo, el trabajo suplementario o de horas extras y las indemnizaciones por despido sin justa causa en los contratos a término indefinido. Igualmente, modificó el contrato de aprendizaje y estableció recursos para el fomento del empleo y protección al desempleado. Estableció programas de microcrédito. Sus disposiciones también tuvieron alcance en materia de seguridad social y en la regulación de las Cajas de Compensación Familiar. La ley se enmarcó en tres pilares: generar más empleo, aumentar el aseguramiento de los trabajadores informales y proteger a quienes no encuentran empleo.

Por otra parte, durante el período analizado también se produjeron otras normas jurídicas que tienen relación con la generación de oportunidades de empleo, la disminución de beneficios económicos laborales o de interés en el campo laboral: ley 361 de 1997 (mecanismos de integración social de personas con limitación), ley 677 de 2001 (tratamientos excepcionales para regímenes territoriales), ley 590 de 2000 (promoción del desarrollo de la micro, pequeña y mediana empresa), ley 1014 de 2006 (fomento a la cultura del emprendimiento). En general, los objetivos de las reformas que se realizaron en materia laboral durante el período analizado estuvieron dirigidos a:

1. Modificar algunos aspectos institucionales del mercado de trabajo: flexibilización de las normas laborales (ley 50 de 1990 y ley 789 de 2002)

2. Ampliar la cobertura del sistema de seguridad social, mejorar la calidad del servicio y hacer viable financieramente el sistema (ley 100 de 1993 y demás normas vinculadas)

3. Proporcionar ayudas directas al empleo, a través del fortalecimiento del apoyo a la creación de empresas (ley 677 de 2001, ley 590 de 2000, ley 789 de 2002, ley 1014 de 2006)

4. Fortalecer la contratación: flexibilizando normas referentes a los contratos y costos laborales, tales como sobrecargos por festivos, jornada de trabajo, disminución de las indemnizaciones (ley 677 de 2001, ley 789 de 2002); estableciendo estímulos (ley 361 de 1997) o como el subsidio temporal al empleo PADE o el régimen especial de aportes (ley 789 de 2002).

5. Mejorar las políticas de inserción laboral: reforma al contrato de aprendizaje (ley 789 de 2002) 
6. Establecer apoyos al desempleo, estableciendo el subsidio del desempleo (ley 789 de 2002)

7.Posibilitar programas para el trabajo en sectores rurales o urbanos, con el fin de ayudar a ciertos trabajadores que tienen dificultades para ingresar o regresar al mercado de trabajo: jóvenes, personas mayores, trabajadores menos capacitados, jefes de hogar (ley 789 de 2002)

¿Qué explica esta tendencia a la flexibilización de los aspectos institucionales que rigen el trabajo? A partir del análisis a los debates de los proyectos presentados en el Congreso en torno a las anteriores leyes, puede inferirse que el contexto económico que llevó a las empresas a competir en un mercado global, la transformación de los modelos de organización empresarial que simplificaron los procesos productivos, y el mal desempeño de los indicadores laborales, fueron factores claves en el impulso de las reformas laborales. Estas reformas se concentraron por tanto en la flexibilización de los aspectos institucionales que rigen el trabajo, bajo el entendido de que éstos creaban costos muy altos y ciertas rigidices que impedían a las empresas generar empleo y ser competitivas. ${ }^{9}$ Por otra parte, al evidenciarse el uso de otras modalidades de trabajo y estar el sistema de seguridad social sustentado sobre la modalidad de trabajo dependiente, se hacia necesario reformar el sistema para garantizar una cobertura a toda la población y a todos los trabajadores, independientemente de la forma en que desarrollen su actividad, para poder cumplir con el compromiso de la cobertura universal.

En efecto, para la justificación de la ley 50 de 1990 se anotó que era necesaria la "adecuación de las leyes del trabajo a la globalización de la economía para expandir la producción, conquistar los mercados internacionales, promover la inversión e incrementar la generación de empleo". Esta reforma se presentó como necesaria para "conciliar lo socialmente deseable con lo económicamente posible, que contribuiría a la estabilidad real de los trabajadores en sus empleos y a la generación de nuevos puestos de trabajo". En la justificación de la ley 100 de 1993 se indicó "la necesidad de esta reforma social dentro del actual proceso de internacionalización de la economía colombiana, que otorgue

\footnotetext{
${ }^{9}$ Este esquema de manejo de la empresa y de la importancia de reducir sus costos, encuadra en un concepto de empresa ligado a la teoría de los costos de transacción. Esto porque bajo tal perspectiva (Alcouffe y Souhaila, 2003) la empresa es una organización que coordina diversas actividades para su funcionamiento y estabilidad. En este sentido, la empresa pretenderá reducir sus costos de transacción, entendidos éstos como los costos que debe pagar cuando acude al mercado. En el mercado el intercambio no es equilibrado y la solución a los problemas que se presenten depende, entre otros factores, de la información y de las elecciones que se tomen. Así, es importante analizar qué tipo de instituciones se establecen, porque éstas estructuran el intercambio y determinan los costos de transacción y de información.
} 
alternativas diferentes, nuevas y opcionales, a los trabajadores colombianos, para garantizar el pago oportuno y cierto de las pensiones". Este proyecto de ley se presentó como un proyecto de ahorro pensional, pero la reforma se hizo a todo el sistema de seguridad social. En la justificación de la ley 789 de $2002^{10}$ se dijo: "tiene como objeto contribuir al fortalecimiento del Estado Social de Derecho mediante medidas trascendentales aunque no de choque que den impulso al mercado laboral y contribuyan a recuperar la confianza tanto de los empleadores como de los trabajadores en la economía colombiana. Urge dinamizar la vida laboral en aspectos que hoy la legislación no facilita y que dentro de márgenes razonables e inspirados en la posibilidad de recuperar espacios para el empleo digno, hagan un poco más atractiva la posibilidad de generarlo e iniciar el camino restaurador de la economía, desde la oportunidad básica de tener acceso al trabajo en condiciones dignas y justas. Para comprender la necesidad de unas medidas de ajuste, es esencial tener clara la magnitud del problema del desempleo y el mercado laboral que enfrenta el país."

Los anteriores argumentos fueron finalmente recogidos en el texto definitivo de la ley 789 de 2002, toda vez que en su artículo 1 se estableció que "el sistema debe crear las condiciones para que los trabajadores puedan asumir las nuevas formas de trabajo, organización y jornada laboral y simultáneamente se socialicen los riesgos que implican los cambios económicos y sociales. Así, el sistema debe asegurar nuevas destrezas a sus ciudadanos para que puedan afrontar una economía dinámica según la demanda del nuevo mercado de trabajo bajo un panorama de crecimiento económico". En conclusión, bien podría afirmarse que las reformas laborales que se produjeron en el período analizado en este artículo estuvieron encaminadas hacia la desregulación o la flexibilización de las relaciones laborales, produciendo una legislación acorde con la descentralización de las relaciones de trabajo dependientes.

\section{Efectos de las reformas en el mercado laboral colombiano: 1990-2006}

El comportamiento de los principales indicadores ${ }^{11}$ del mercado laboral durante el período analizado fue el siguiente: La tasa de ocupación o de empleo, que en 1990 era del 51,4\%, al final del año 2000 se situó en 51,9\%; a

${ }^{10}$ Para la aprobación de esta ley fue importante el concepto 3199 del 30 de septiembre de 2002.

La finalidad de dicho concepto fue hacer un diagnóstico del mercado laboral al CONPES

(Consejo Nacional de Política Económica y Social) y someter a su consideración una serie de políticas encaminadas a aliviar el problema del desempleo en el país. Así, se propusieron ajustes institucionales en materia de recargos, indemnizaciones, aportes parafiscales, salarios para trabajadores nuevos, entre otros.

${ }^{11}$ Conforme a las estadísticas del DANE. Ver gráficas y glosario anexos. Para el período 1990-1998 
comienzos de 2001 se ubicó en 52,4\%, y a inicios de 2006 se ubicó en 51,4\%. Por su parte, la tasa de desempleo ascendió de 10,2\% en 1990 a 19,7\% a finales del año 2000; en el año 2001 se ubicó en 15,1\% y en 2006 se ubicó en 12,1\%. La tasa de subempleo ${ }^{12}$ aumentó de $12,9 \%$ en 1991 a 28,7\% en el año 2000; en el año 2001 ascendió hasta 31,6\% y en 2006 se situó en 33,8\%.

La tasa de crecimiento del empleo pasó de 4,6\% en 1992 a -0,90\% en 1996. Presentó una recuperación a 4,4\% en 1997, para volver a caer a - - 2,25\% en 1999. En el año 2000 comienza nuevamente una recuperación de esta tasa y se sitúa en 4,7\%. En 2001 la tasa de crecimiento del empleo se ubicó en 1,33\%, ascendió a 4,8\% hacia finales de 2003; en el año 2005 presenta una mejoría y decreció a - 0,53\% en el 2006. La participación del sector público en la tasa de ocupación pasó de 9,63\% en 1991 a 6,13\% en el año 2005.

El sector informal ${ }^{13}$ pasa de $54 \%$ en 1992 a $60 \%$ en el año 2000. Por su parte el sector formal desciende de 46\% en 1992 a 40\% en el año 2000. Del total de ocupados en el año 2001, el 60\% pertenece al sector informal y el $40 \%$ al sector formal. Para el año 2006, del total de ocupados, el 58\% pertenece al sector informal y el $42 \%$ al sector formal. Según la posición ocupacional, puede observarse que en el sector informal, el trabajador por cuenta propia es la tendencia predominante durante todo el período objeto de estudio, seguido por el empleado particular, el empleado doméstico y el trabajador familiar sin remuneración. En el sector formal, como es lógico, el empleado particular es la tendencia predominante, frente al empleado del gobierno y el trabajador por cuenta propia durante todo el período analizado.

el DANE aplicaba la ENH (encuesta nacional de hogares) a siete áreas metropolitanas. A partir de enero de 1999 el DANE implementó el proyecto de ECH (encuesta continúa de hogares) en la ciudad de Bogotá y otras áreas metropolitanas. Desde enero de 2000, la ECH se aplica a trece áreas metropolitanas. A partir de abril de 2001 el DANE hizo modificaciones sustanciales en algunas de las definiciones que sirven de fundamento a algunos puntos de la ECH. En junio de 2006 nuevamente el DANE introdujo cambios en la metodología para la recolección de información. Aunque los anteriores cambios en definiciones y metodologías por parte del DANE han generado fuertes críticas de parte de analistas del mercado laboral, quienes cuestionan algunos datos, dicha entidad ha realizado diferentes análisis sobre la comparabilidad de las series de encuestas anteriores a julio de 2006.

${ }^{12}$ Actualmente el DANE utiliza la tasa de subempleo bajo dos categorías: objetivo y subjetivo. El subempleo objetivo indica que la persona tiene el deseo de mejorar su empleo y ha hecho una gestión para materializar su aspiración y está en disposición de efectuar el cambio. El subempleo subjetivo indica que la persona tiene el simple deseo de mejorar sus ingresos, tener un mayor número de horas de trabajo o de tener una labor más apropiada de acuerdo con sus competencias laborales.

${ }^{13}$ Los datos sobre informalidad y subempleo pueden tener cierta variación frente al comportamiento real del mercado laboral, dadas las definiciones utilizadas por el DANE para agrupar en estas categorías a ciertos grupos de trabajadores y por el componente subjetivo que tienen ciertas

\section{PRECEDENTE}


En relación con la afiliación al sistema de seguridad social, para el período 2001-2006, al inicio y al cierre del período, la situación es la siguiente: en la población informal, la afiliación a salud pasa de $56 \%$ a $74 \%$; en pensiones está por debajo de 10\% en todo el período. En el sector formal, la afiliación a salud pasa de $91 \%$ en 2001 a 94\% en el año 2006. En pensiones, se mantiene alrededor del $70 \%$.

$\mathrm{El}$ análisis de los anteriores indicadores permite indicar que la composición del empleo durante el período analizado fue la siguiente: reducción del empleo público y privado (trabajo dependiente), aumento considerable del sector informal y del trabajo independiente. Asimismo, hay un bajo aumento de la tasa de crecimiento del empleo, bajo descenso de la tasa de desempleo y aumento de la de subempleo. El crecimiento del sector informal explica en parte el bajo nivel de afiliación de trabajadores al sistema de seguridad social, porque un componente del tipo de trabajo en dicho sector corresponde con bajos niveles de cumplimiento de las normas laborales y de seguridad social, aunque no puede generalizarse.

En relación con la composición de la población ocupada según la rama de actividad económica se evidencia un crecimiento de la actividad en el sector del comercio y en el de servicios comunales, sociales y personales. Esta última actividad es una rama muy heterogénea; además de incluir la administración pública y algunas ocupaciones altamente calificadas, por ejemplo en la salud y educación, comprende toda una serie de servicios de carácter informal como el servicio doméstico, las actividades de esparcimiento y los servicios personales. Por tanto, los servicios comunales, sociales y personales, contienen un componente de informalidad laboral, aunque no se pueden igualar sector servicios e informalidad, porque no siempre el desplazamiento de las actividades hacia el sector servicios implica un trabajo en condiciones inadecuadas. Esto sugiere que sería conveniente revisar el concepto de informalidad y determinar qué tipo de trabajos o bajo cuáles condiciones serían agrupados en este concepto.

Lo anterior significa que algunas de las tendencias que se describieron en la primera parte sobre las transformaciones del trabajo se evidencian en el contexto colombiano. Las prácticas laborales cada vez utilizan menos la modalidad de trabajo dependiente (empleo) y acuden principalmente a las modalidades de trabajo independiente y asociativo. La descentralización de la actividad

preguntas en el instrumento utilizado para la medición (Núñez, 2002; Urrutia, 2002). Asimismo, no debe olvidarse que durante el período analizado se presentó un auge en la creación de organizaciones de trabajo asociado que ofrecían mano de obra a bajo costo a algunos sectores empresariales del país. Por esto, gran parte de los trabajadores de las organizaciones de trabajo asociado están asociados al sector informal. Fue tal el uso indebido de tales organizaciones que a partir del año 2004 el gobierno nacional se vio obligado a expedir una serie de reglamentaciones para adecuar dichas organizaciones a las disposiciones legales. 
productiva de la empresa estimula este último tipo de modalidades. Además, el proceso de simplificación del proceso productivo hace que la empresa no siempre requiera realizar contrataciones de tipo laboral para los servicios que demanda. Esto explica la reducción del trabajo dependiente. ${ }^{14}$ De ahí que el nivel de crecimiento de la tasa de empleo puede estar más asociado con el crecimiento de actividades en el sector servicios que con el crecimiento del trabajo dependiente formal (empleo). Esto significa que las empresas han generado trabajo más que empleo y conforme a los servicios que demandan.

Por otra parte, se podría sugerir que la vía tomada por las reformas laborales para estimular la generación de empleo no tuvo el resultado esperado porque la forma en que se organiza la actividad productiva en las empresas en el contexto actual hace un uso mayor de otras modalidades de trabajo (asociativa e independiente) frente a un menor uso de la modalidad de trabajo dependiente (celebración de contratos de trabajo). Las reformas laborales se concentraron en el estímulo a la modalidad de trabajo dependiente al flexibilizar diferentes aspectos que tienen relación con las obligaciones derivadas de un contrato de trabajo. Bajo esta perspectiva, si los desempleados no se han beneficiado en la forma esperada como consecuencia de las reformas laborales, se podría afirmar que los trabajadores ocupados a través de un contrato de trabajo resultaron afectados con tales reformas porque algunas de las garantías o beneficios económicos fueron reducidos con ellas.

En conclusión, a pesar de que las normas laborales se flexibilizaron y se han ido acondicionando a un nuevo modelo de organización empresarial, otorgando importantes estímulos para la generación de empleo, sus resultados sobre el empleo (trabajo dependiente) no fueron los esperados y por el contrario posibilitaron una tendencia al crecimiento del sector informal. Para Lora y Márquez (1998) el comportamiento del mercado laboral obedeció principalmente a tres razones: cambios en la organización productiva en la empresa, políticas macroeconómicas ${ }^{15}$ y flexibilización de las normas laborales. Estos mismos factores fueron los responsables de la expansión del sector informal. Eduardo

${ }^{14}$ De ahí el cuestionamiento que se le hace a la legislación laboral en el sentido de que no está otorgando protección a las personas que pretende proteger (Pages, 2004; Pages y Saavedra, 2002).

${ }^{15}$ Las políticas macroeconómicas también incidieron en el mercado laboral ya que el crecimiento del empleo es uno de los objetivos de dichas políticas. La macroeconomía es el estudio del conjunto de la economía que se ocupa de cuestiones tales como el crecimiento económico, el desempleo, la inflación, y las políticas públicas que pueden afectar toda la actividad económica. Los objetivos fundamentales del estudio de la macroeconomía son tres: crecimiento económico, un nivel de empleo elevado y la estabilidad de los precios. Cada uno de estos factores se encuentra interrelacionado entre sí. Dado que este artículo establece una relación entre los cambios en la organización productiva y la regulación laboral, no considero necesario hacer un balance macroeconómico del período analizado. No obstante pueden verse trabajos importantes en:

\section{PRECEDENTE}


Lora y Gustavo Márquez (1998) lo explican así: "las reformas estructurales han alterado la composición de la demanda laboral debido a que han abaratado el costo de uso de capital y acelerado el proceso tecnológico. Los efectos han sido una expansión del empleo informal y del sector servicios" En otro estudio, Eduardo Lora y Mauricio Oliveira (1998) indicaron que "la informalidad se explica debido a las características de los empleos en el sector servicios y a la caída relativa en la demanda de mano de obra no calificada. La demanda laboral se ha desplazado hacia el trabajo calificado debido a la adopción de nuevas tecnologías". Esto ha generado fuertes diferencias salariales entre el trabajador calificado y el no calificado.

En consecuencia, el bajo aumento de la tasa de crecimiento de empleo, el bajo descenso de la tasa de desempleo, el aumento de la tasa de subempleo y la fuerte expansión del sector informal permiten cuestionar los criterios económicos que orientaron las reformas laborales en función del mercado, bajo el entendido de que corrigiendo las rigideces que generan ciertas disposiciones jurídicas se lograría un mejor funcionamiento del mercado laboral y, por lo tanto, un crecimiento del empleo. ${ }^{16}$ Esto no implica que tales criterios no puedan tener validez, lo que se cuestiona es que las reformas laborales se hayan centrado en ellos.

\section{La importancia del diálogo interdisciplinario en torno al tema laboral}

Los efectos de las reformas mencionadas sobre el mercado laboral sugieren que los problemas en torno a la regulación del trabajo son asuntos complejos que no conviene abordar desde una sola disciplina. Las dinámicas del campo laboral dependen de múltiples variables, las cuales son objeto de estudios de diferentes disciplinas, entre ellas la economía, la sociología o la historia. Los problemas que se presentan en el campo laboral no pueden reducirse a

Echeverri, 1999; Arango y Posada, 2003; Cárdenas y Urrutia, 2004; Cabrera 2005; Banco de la República, 1999, 2005.

${ }^{16}$ Precisamente, uno de los cuestionamientos mas recientes tiene relación con la última reforma introducida a través de la ley 789 de 2002. Esta ley ha generado diferentes trabajos de investigación desde diversos sectores (Observatorio del Mercado laboral y la Seguridad Social de la Universidad Externado, 2003, 2004; Gaviria, 2004; ENS, 2005; López, Rhenals, Castaño, 2005; Núñez, 2005). Diferentes comentarios podrían hacerse a cada una de estas investigaciones a partir de las metodologías utilizadas y de los resultados obtenidos, pero lo cierto es que es difícil asociar el crecimiento del empleo a las reformas introducidas por dicha ley. Estos estudios cobran mayor importancia porque la mencionada ley creó una comisión de verificación para evaluar los efectos de la reforma laboral sobre el empleo. Del trabajo de la comisión se conoce poco. El gobierno avaló un estudio publicado por el Ministerio de la Protección Social en el que se concluye que la reforma tuvo impactos favorables sobre el trabajo. 
la existencia de un mayor o menor número de normas, ni tampoco a la idea de que una disciplina en particular puede resolver por sí sola tales problemas (Cardona y otros, 2004). La importancia de los diferentes estudios que desde diversas disciplinas se han hecho sobre el tema laboral no sólo radica en el valioso aporte analítico acerca de los problemas relacionados con el trabajo y el empleo sino, también, en las serias inquietudes que le han planteado al derecho sobre la función que tiene la regulación en el desempeño de los mercados laborales y la necesidad, por tanto, de una normatividad que optimice la relación entre las prácticas laborales y las normas que las regulan.

Es por lo tanto importante establecer un diálogo entre el derecho y otras disciplinas para abordar problemas comunes en torno al trabajo. Este camino se justifica por dos razones. La primera, porque las reformas laborales no han logrado, no al menos con las cifras esperadas, el crecimiento del empleo. La segunda justificación tiene relación con el tema del incumplimiento de la legislación laboral. Se ha anotado que un componente del trabajo en el sector informal está asociado a bajos niveles de cumplimiento de las normas laborales y de seguridad social. Si bien es un hecho notorio que existen escenarios en los cuales la regulación laboral no se cumple, no existe una adecuada información del por qué o de las causas de este fenómeno. Si la economía ha llamado la atención sobre los efectos que la regulación genera en el funcionamiento del mercado laboral, se hace necesario investigar, desde una perspectiva interdisciplinaria, la relación que establecen los individuos (empleadores y trabajadores) con las instituciones que regulan el mercado laboral, para así tener mayores elementos para comprender mejor el papel que desempeñan las instituciones en dicho mercado y su eficacia. De esta forma, el estudio de la eficacia de las instituciones sería el marco que permitiría el diálogo entre el derecho y otras disciplinas, o entre el derecho y la economía. La apropiación de la teoría neoinstitucionalista constituiría un enfoque teórico que, en principio, permitiría abordar el estudio de la eficacia de las instituciones. La importancia de esta teoría radica en que ha "hecho hincapié en la enorme influencia que tienen los arreglos institucionales como presupuestos de funcionamiento de los mercados, que es una forma de reconocer, desde el campo económico, la importancia del derecho y de las instituciones en los procesos económicos" (Uprimny, 2006).

El neoinstitucionalismo, conforme a Douglas North, uno de sus principales exponentes, indica que existe una relación entre las instituciones y el crecimiento económico. Propone una explicación de las instituciones basada en una teoría de la conducta humana, pero rechaza la idea del actor racional que existe en la economía clásica. Las instituciones "forjan el esqueleto de la interacción humana al proporcionar una estructura de incentivos como guía del comportamiento humano" (North, 2007). Ellas determinan la estructura

\section{PRECEEENTE}


del intercambio que determinan los costos de transacción y de información. Para el neoinstitucionalismo existen unas instituciones formales (producidas y generadas por el Estado)y otras informales (producidas espontáneamente por la población) que se constituyen en las "reglas de juego" y guían el comportamiento de los individuos. En este sentido, las primeras son fáciles de cambiar; las segundas no. Así, la eficacia de las instituciones formales depende de su relación con las informales. Por otra parte, las organizaciones y los individuos tratarán de cambiar las instituciones cuando los costos de transacción (costos en que se incurre cuando se acude al mercado) son altos, aunque las relaciones de poder pueden impedir dicho cambio. Si tales costos son muy altos, las instituciones formales no van a ser utilizadas y se utilizarán más las instituciones informales o se crearán otras de este mismo tipo.

De esta forma, dado que el neoinstitucionalismo propone una explicación de las instituciones en relación con la conducta humana, el tema del incumplimiento de la legislación, al igual que la tendencia de crecimiento del sector informal del mercado laboral y su relación con el sector formal, podrían ser analizadas desde aquella teoría por la economía y el derecho. La historiografía podría contribuir a este diálogo multidisciplinar al ofrecer referentes teóricos propios de esta disciplina que permitan un estudio más amplio de las instituciones: ¿cuáles eran las condiciones sociales, económicas y políticas que posibilitaron el surgimiento de las instituciones? icómo se han transformado las instituciones? icuál ha sido la incidencia del Estado y de los actores sociales en esa transformación? iqué papel han jugado las disciplinas en esa transformación? Los resultados de tales investigaciones podrían enriquecer la discusión académica sobre la regulación laboral y el funcionamiento del mercado de trabajo. Si bien el neoinstitucionalismo ha recibido agudas críticas desde diversos campos y en algunos de sus exponentes presenta una visión instrumentalista del derecho que es preciso matizar, algunas corrientes y aspectos de esta teoría podrían ser importantes para estimular el diálogo entre economistas y abogados.

Este trabajo conjunto entre la economía y el derecho a partir de problemas y enfoques comunes permitiría superar la disputa que se ha dado entre ambas disciplinas por lograr la "autoridad" en el campo científico ${ }^{17}$ (Londoño 2002; Uprimny, 2006). Y para esto, como lo advierte Jaime Eduardo Londoño (2004), este diálogo debe realizarse en las zonas de fronteras de ambas disciplinas y

\footnotetext{
${ }^{17}$ El concepto de campo científico corresponde al término empleado por el sociólogo Pierre Bourdieu (2003), para quien "el campo científico, al igual que otros campos, es un campo de fuerzas dotado de una estructura, así como un capo de luchas para conservar o transformar ese campo de fuerzas. (...) El capital científico es un tipo especial de capital simbólico, capital basado en el cocimiento y en el reconocimiento. Este poder, que funciona como una forma de crédito,
} 
no desde el concepto de campo científico, porque en este último "es difícil construir un diálogo entre ambas disciplinas ya que significaría una pérdida de posiciones de poder y, por tanto, de autoridad científica". Las denominadas zonas de frontera "son zonas de alteridad, de encuentro y desencuentro, de interacción y de conflicto" en las que puede haber "diálogo, contacto, interacción, conflicto, transición y negociación”.

\section{Retos del derecho laboral frente a la transformación del trabajo}

Los procesos de transformación del trabajo en la década de los 90 del siglo XX estuvieron determinados por el contexto económico, el avance de la tecnología y las comunicaciones y las teorías de reorganización administrativa de la empresa y del trabajo. Dichos contextos evidencian cómo los cambios en la organización productiva posibilitaron "una evolución del pensamiento político hacia una mayor confianza en el papel de los mercados y la disminución de la acción del Estado" (OIT, 2006). Así, el interés por lograr un mejor desempeño del mercado laboral generó una serie de reformas a los aspectos institucionales que lo rigen (normas laborales). Estos procesos han generado efectos positivos y negativos en el campo laboral. Asimismo, han transformado el contenido y la forma misma de trabajo. No obstante, debe indicarse que el modelo de organización del trabajo que era el predominante antes de la década de los 90 no ha desaparecido, por el contrario, permanece junto a nuevas formas de organización. En efecto, las formas de organización del trabajo se concretan en dos usos y prácticas de los empleadores y de los trabajadores. La primera de ellas, las prácticas del trabajo dependiente, sustentada en las normas tradicionales de la legislación laboral sobre protección del trabajo. La segunda, la de impulso de otras modalidades de trabajo, principalmente el independiente y el

supone la confianza o la fe de los que lo soportan porque están dispuestos (por su formación y por el mismo hecho de la pertenencia al campo) a conceder crédito y fe. (...) El capital científico funciona como un capital simbólico de reconocimiento que circula primordialmente, y, a veces, de manera exclusiva, dentro de los límites del campo (aunque puede ser reconvertido en otros tipos de capital, especialmente económico): el peso simbólico de un científico tiende a variar de acuerdo con el valor distintivo de sus contribuciones y la originalidad que sus colegas-competidores reconocen a su aportación distintiva." Para Bourdieu (1990) el campo es un espacio social específico en donde suceden una serie de interacciones. Es un sistema particular de relaciones objetivas que pueden ser de alianza o conflicto, de concurrencia o de cooperación entre posiciones diferentes, socialmente definidas e instituidas, independientemente de la existencia física y de los agentes que lo ocupan. Las posiciones que asuman en el campo dependen de los capitales. Éstos son lo recursos que tienen los actores para "jugar” en el campo. Pueden ser: económico, cultural, social, simbólico, científico 
asociativo, que rige las prácticas y las actividades productivas contemporáneas, caracterizadas por la flexibilización, la movilidad y la especialización. Estas últimas prácticas están redimensionando la noción de empleo.

Las reformas laborales que se produjeron en Colombia en la década de los 90 estuvieron orientadas a flexibilizar o desregularizar la legislación laboral con el fin de promover la generación de empleo y lograr una cobertura del sistema de seguridad social. Si bien los esfuerzos de la legislación se orientaron a estimular el ejercicio del trabajo bajo diversas modalidades, las reformas se concentraron principalmente en la generación de estímulos para la modalidad de trabajo dependiente por medio de la flexibilización del contrato de trabajo. Sin embargo, las prácticas laborales en las empresas se han orientado a promover otras modalidades de trabajo, tales como la independiente y la asociativa. En este hecho se podría encontrar una explicación a por qué las reformas laborales no han tenido los efectos deseados. A esto se debe agregar que la simplificación de los procesos productivos ha estimulado la demanda de servicios por fuera del contrato laboral o de las relaciones laborales dependientes.

La comprensión de este proceso de transformación del trabajo impone retos al derecho laboral. El derecho del trabajo debería por tanto revisar el concepto de subordinación jurídica, la cual no debería estar hoy en día definida en términos exclusivamente del contrato de trabajo. La tendencia al uso de otras modalidades de trabajo diferentes al trabajo dependiente justifica esta redefinición. Pero debe tenerse presente que tal redefinición jurídica no implica abrir a las demás modalidades del trabajo la regulación tradicional del derecho laboral y vinculada al contrato de trabajo. Implica pensar en unos estándares mínimos que garanticen unos principios básicos de protección al trabajo, independiente de la modalidad de trabajo bajo la cual se realice. No está de más recordar que la Constitución Política establece una protección especial al trabajo en todas sus modalidades. La Constitución, al considerar al trabajo como un valor, un principio fundante del Estado Social de Derecho y un derecho fundamental, impone al Estado el diseño de políticas públicas que contengan una efectiva protección de la dignidad, los derechos y la libertad de los trabajadores, independientemente de la modalidad de trabajo bajo la cual desarrollen su actividad.

Por otra parte, Colombia es miembro de la Organización Internacional del Trabajo (OIT) y este solo hecho obliga a los Estados miembros a orientar su regulación conforme a las disposiciones de dicho organismo. La Constitución Política colombiana refuerza este mandato al establecer que las normas internacionales que se refieran a derechos humanos - entre las que se encuentran algunos convenios de la OIT - prevalecen sobre las normas constitucionales y cumplen efectos interpretativos sobre las demás normas del ordenamiento jurídico (Art. 93). La OIT no se ha ocupado sólo del trabajo dependiente, por 
el contrario, ha desarrollado directrices importantes para el trabajo realizado bajo otras modalidades, como el independiente y el asociativo. Esto implica que la aplicación de las normas internacionales del trabajo en tales campos debe ser fortalecida en nuestro ordenamiento jurídico.

La anterior redefinición del derecho laboral se justifica porque el principio que funda la legislación laboral requiere, para su correcto y efectivo funcionamiento, que éste se construya sobre las condiciones sociales y económicas que determinan las actividades productivas. Tradicionalmente el discurso de la norma laboral se ha basado en la pretensión de posibilitar, regular o garantizar un equilibrio entre los intereses de empleadores y los de los trabajadores. Este objetivo debe mantenerse. $\mathrm{Y}$ es por esto que es necesario reflexionar sobre la ampliación de los sujetos regulados por el derecho laboral, sin que esto implique, como ya se anotó, que la eventual regulación de otras modalidades laborales se realice de la misma forma como hasta ahora se ha configurado la legislación laboral tradicional, es decir, sobre la base de un contrato de trabajo. ${ }^{18}$ Bajo los anteriores presupuestos, y teniendo presente el modelo actual de organización del trabajo, la agenda de la legislación laboral podría concentrarse en los siguientes aspectos:

1. Desarrollar el mandato establecido en el artículo 53 de la Constitución Política de expedir un nuevo estatuto del trabajo que respete los principios fundamentales constitucionales. Este estatuto se aplicaría a todas las modalidades de trabajo (dependiente, independiente, asociativo y voluntario) conforme a lo establecido en el artículo 25 de la Constitución. Se trataría de un estatuto que, como lo indica el programa de trabajo decente de la OIT, preste mayor atención a la vulnerabilidad de los trabajadores, a las condiciones de trabajo y a la calidad del empleo.

2. Redefinir el concepto de subordinación jurídica.

3. Fortalecer los mecanismos de control para la verificación del cumplimiento de las normas laborales.

4. Revisar y/o fortalecer los programas de protección para ciertos grupos de trabajadores que demandan una mayor atención (trabajadores de la construcción, trabajadores domésticos, trabajadores independientes).

\footnotetext{
${ }^{18}$ En el derecho comparado se encuentran alternativas interesantes de regulación. En SánchezCastañeda (2005) pueden encontrarse algunos aspectos que han sido regulados por el derecho italiano y el alemán en el caso del trabajo independiente.
} 


\section{Bibliografía}

Alcouffe, Alain y Kammoun Souhaila (2003), "Enfoque económico de las competencias de la firma. Hacia una síntesis de las teorías neoinstitucionales y evolucionistas", Boletín No. 154. Montevideo, Cinterfor. en: http://www. oitcinterfor.org/public/spanish/region/ampro/cinterfor/publ/catalogo/autor/ alco/index.htm

Arango, Luís Eduardo y Carlos Esteban Posada (2003), "La participación laboral en Colombia”, en: Coyuntura social, No. 28, junio.

Banco de la República (2005), "Un vistazo a la economía, tercer trimestre de 2004", en: Revista del Banco de la República.

(1999), "Memorando de políticas económicas", en: Revista del Banco de la República, Vol. LXXII, No. 866, diciembre de 1999.

Beck, Ulrich (2000), Un nuevo mundo feliz. La precariedad del trabajo en la era de la globalización, Paidós, Barcelona.

Banco Interamericano de Desarrollo (2004), Se buscan buenos empleos. Los mercados laborales en América Latina. Banco Interamericano de Desarrollo, Washington, D.C.

Boltanski, Luc y Eve Chiapello (2002), El nuevo espíritu del capitalismo, Ediciones Akal, Madrid.

Bourdieu, Pierre (2003), El oficio de científico. Ciencia de la ciencia y reflexividad, Curso del College de France 2000-2001, Anagrama, Barcelona.

Traducción de Martha Pou.

(1990), Sociología y cultura, Editorial Grijalbo, México.

Cabrera, Mauricio (2005), "Políticas macroeconómicas para el bienestar", en: Revista Economía y Desarrollo, Universidad Autónoma de Colombia, Vol. 4, No. 2, septiembre.

Carnoy, Martín (2001), El trabajo flexible en la era de la información, Alianza Editorial, Madrid.

Cárdenas, Mauricio y Miguel Urrutia (2004). "Impacto social del ciclo económico en Colombia: 1989- 2004”, en: Coyuntura social, No. 30, junio de 2004.

Cardona Rubert, María Belen, Juan López Gandía, Frederic López i Moora, Josep Vicent Saragossá i Saragossá (2004), Derecho del empleo, Tirant lo Blanch, Valencia.

Carnoy, Martin (2001), El trabajo flexible en la era de la información, Alianza Editorial, Madrid.

Capella, Juan Ramón (1997), Fruta prohibida, Trotta, Madrid.

Castel, Robert (2004), La inseguridad social. ¿Qué es estar protegido?, Manantial, Buenos Aires. 
Castells, Manuel (2001). La galaxia Internet. Reflexiones sobre Internet, empresa y sociedad, Ediciones Areté, Madrid.

(1999). La era de la información: economía, sociedad y cultura. La sociedad red, Siglo XXI Editores, México.

Castillo, Juan José (1998), A la búsqueda del trabajo perdido, Editorial Tecnos S.A., Madrid

Coriat, Benjamin (2001), El taller y el cronómetro: ensayo sobre el taylorismo, el fordismo y la producción en masa, Siglo XXI de España Editores, Madrid.

(2000), Pensar al revés: trabajo y organización en la empresa japonesa, Siglo XXI de España Editores, Madrid.

De la Garza Toledo, Enrique (2001), El trabajo del futuro. El futuro del trabajo, Clacso, Buenos Aires.

Deveali, Mario L. (1966), Tratado de derecho del trabajo, 5 volúmenes, la Ley, Buenos Aires.

Dombois, Rainer y Carmen Marina López (1993), Cambios técnicos, empleo y trabajo en Colombia, Fescol, Bogotá.

Echeverry Garzón, Juan Carlos (1999), "La recesión de fin de siglo en Colombia: flujos, balances y política anticíclica”, en: Planeación y Desarrollo, Vol. XXX, No. 2 abril- junio 1999.

Escuela Nacional Sindical (2005), ¿Qué ha pasado con el empleo dos años después de la reforma laboral?, Investigación dirigida por Hernán Vásquez Fernández, ENS, Bogotá.

Gaviria, Alejandro (2004), "La ley 789 de 2002: ifuncionó o no?", Centro de Estudios sobre Desarrollo Económico, Documento 2004-45, Facultad de Economía - Universidad de los Andes, Bogotá.

Guasch, J. Luis (1998), Reforma laboral y creación de empleo: la agenda incompleta de los países de América Latina y el Caribe, CIEDLA, Buenos Aires.

Londoño Motta, Jaime Eduardo (2004), "Neoinstitucionalismo: en la encrucijada del proceso de apropiación por las ciencias sociales en Colombia", en: Precedente, Facultad de Derecho y Ciencias Sociales, Universidad Icesi, Cali. (2002), "Neoinstitucionalismo: ies posible un diálogo entre historiadores, juristas y economistas?", en: Precedente, Anuario jurídico 2002, Facultad de Derecho y Humanidades, Universidad Icesi, Cali.

López Castaño, Hugo, Remberto Rhenals y Elhin Castaño (2005), "Impacto de la reforma laboral sobre la generación y calidad del empleo”, Cuaderno No. 1 de política social. Ministerio de la Protección Social, Bogotá.

Lora, Eduardo y Olivera Mauricio (1998), Memorias del seminario ¿Cuál es el problema del empleo de América Latina y cómo enfrentarlo?, Banco Interamericano de Desarrollo, Cartagena.

Lora, Eduardo y Gustavo Márquez (1998), Memorias del seminario iCuál es

\section{PRECEDENTE}


el problema del empleo de América Latina y cómo enfrentarlo?, Banco Interamericano de Desarrollo, Cartagena.

Martín Artiles, Antonio (1995), Flexibilidad y relaciones laborales. Estrategias empresariales y acción sindical, Consejo Económico y Social, España.

North, Douglas (2007). Para entender el proceso de cambio económico, Grupo editorial norma - Facultad de Economía, Universidad de los Andes, (2005 primera edición), Bogotá.

Novick, Marta y Martina Miravalles (2002), La dinámica de oferta y demanda de competencias en un sector basado en el conocimiento en Argentina, CEPAL, Chile.

Novick, Marta, Marta Bartolomé, Mariana Buceta, Martina Miravalles y Cecilia Senen (1998), Nuevos puestos de trabajo y competencias laborales. Un análisis cualitativo en el sector metalmecánica argentino, OIT, Montevideo.

Núñez Méndez, Jairo Augusto (2005), "Éxitos y fracasos de la reforma laboral en Colombia", Centro de Estudios sobre Desarrollo Económico, Documento No. 2005-43, Facultad de Economía Universidad de los Andes, Bogotá.

(2002), "Empleo informal y evasión fiscal en Colombia”, Archivos de economía, Documento 210, 29 de octubre de 2002, Departamento Nacional de Planeación, Bogotá.

Observatorio del Mercado de Trabajo y la Seguridad Social (2003), "La ley 789 de 2002. Reforma laboral colombiana”, Boletín número 6, Universidad Externado de Colombia, Bogotá.

(2004), "Seguimiento a la ley 789 de reforma laboral. Año 2003", Cuaderno de trabajo No. 5., Universidad Externado de Colombia, Bogotá.

OIT (2006), "Cambios en el mundo del trabajo", Memoria del director general, Conferencia internacional del trabajo, 95ª reunión. Oficina Internacional del Trabajo, Ginebra.

Pages, Carmen (2004), Se buscan buenos empleos. Los mercados laborales en América Latina, BID - Alfaomega, Bogotá.

Pages, Carmen y Jaime Saavedra (2002). Legislación laboral: algunos principios fundamentales, evidencia empírica y políticas en América Latina.

Sánchez-Castañeda, Alfredo (2005), "El derecho del trabajo en constante transformación: de la disminución a la ampliación del estatus del sujeto laboral regulado", en: Kurczyn, Patricia (coord.) Derecho social. Memoria del congreso internacional de culturas y sistemas jurídicos comparados, Universidad Nacional Autónoma de México, México D.F.

Sennett, Richard (2000), La corrosión del carácter. Las consecuencias personales del trabajo en el nuevo capitalismo, Anagrama, Barcelona.

Uprimny Yepes, Rodrigo (2006), "Legitimidad y conveniencia del control 
constitucional a la economía", en: ¿Justicia para todos? Sistema judicial, derechos sociales y democracia en Colombia, Grupo editorial norma, Bogotá.

Urrea, Fernando (2003), "Una mirada sociológica al "outsourcing”: las tendencias de la flexibilidad externa e interna y la empresa red en el capitalismo contemporáneo”. Memorias, ACRIP, Manizales.

(2002), "Globalización y prácticas de flexibilización laboral en grandes empresas: el caso colombiano", en: Sociología del trabajo, nueva época, número 44, invierno de 2001-2002. Madrid.

Urrea, Fernando y Carlos Alberto Mejía (2000), "Innovación y cultura de las organizaciones en el Valle del Cauca", en: Innovación y cultura de las organizaciones en tres regiones de Colombia. Varios autores, Colciencias y Corporación Calidad, Tercer Mundo Editores, Bogotá.

Urrutia Montoya, Miguel (2002), "El subempleo en Colombia", Nota editorial. en: Revista del Banco de la República, No. 898, agosto de 2002, Banco de la República, Bogotá.

Weiis, Anita (1994), La empresa colombiana entre la tecnocracia y la participación. Del taylorismo a la calidad total, Universidad Nacional de Colombia, Bogotá.

\section{GLOSARIO}

TGP. Tasa global de participación. Es la relación porcentual entre la población económicamente activa y la población en edad de trabajar. Este indicador refleja la presión de la población en edad de trabajar sobre el mercado laboral.

TO. Tasa de ocupación o de empleo. Es la relación porcentual entre la población ocupada y el número de personas que componen la población en edad de trabajar (PET).

TD. Tasa de desempleo. Es la relación porcentual entre el número de personas que están buscando trabajo y el número de personas que integran la fuerza laboral (PEA).

TS. Tasa de subempleo. Es la relación porcentual de la población ocupada que quiere y puede trabajar más horas a la semana, o en otro empleo, y el número de personas que integran la fuerza laboral (PEA).

Ocupación. Es el oficio o profesión (cuando se desempeña en ésta) de una persona, independiente del sector en que puede estar empleada, o del tipo de estudio que hubiese recibido. 
Ocupados. Son las personas que participan en el proceso de producción de bienes y servicios.

Rama de actividad. Es la actividad a la cual se dedica la empresa, fábrica, finca o taller, donde trabaja la persona.

Posición ocupacional. Es la posición que la persona adquiere en el ejercicio de su trabajo, ya sea como asalariado, obrero o empleado de empresa particular, obrero o empleado del gobierno, empleado doméstico como trabajador por cuenta propia, empleador o como trabajador familiar sin remuneración.

Subempleo. Es la población compuesta por personas ocupadas que quieren y pueden trabajar más tiempo, o desean otro empleo. Puede ser por insuficiencia de horas, es decir, aquellas personas ocupadas que desean trabajar más horas, están disponibles para hacerlo y han trabajado menos de la jornada legal (48 horas semanales), o por condiciones de empleo inadecuado. Se considera que una persona ocupada tiene condiciones de empleo inadecuado cuando ejerce una actividad que no se ajusta a su profesión o habilidades (subempleo por competencias), o cuando los ingresos laborales son insuficientes para atender sus gastos normales (subempleo por ingresos).

Desocupados. Son las personas de la PEA que están sin empleo, están en busca de uno y están disponibles para empezar a trabajar. Pueden ser cesantes o aspirantes. Los primeros son las personas que, habiendo trabajado antes, se encuentran desocupadas. Los segundos, son las personas que están buscando trabajo por primera vez.

Sector informal. Operativamente se consideran trabajando en el sector informal las personas que cumplan las siguientes características: empleados particulares y obreros que laboren en establecimientos, negocios o empresas que ocupen hasta diez personas en todas sus agencias y sucursales; trabajadores familiares sin remuneración; empleados domésticos; trabajadores por cuenta propia, excepto los independientes profesionales y los empleadores de empresas de diez trabajadores o menos. 